\title{
Conservative Treatment of Keratocystic Odontogenic Tumour:-A Case Report
}

\author{
Dr. Rajesh Khirsagar ${ }^{1}$, Dr. Nitin. J. Oswal ${ }^{2}$, Dr. Vikram Singh ${ }^{3}$, Dr. Pratik Raut ${ }^{4}$ \\ ${ }^{I}$ (Department Of Oral And Maxillofacial Surgery/ Bharati Vidyapeeth Deemed University, Dental College And \\ Hospital, Pune, India) \\ ${ }^{2}$ (Department Of Oral And Maxillofacial Surgery/ Bharati Vidyapeeth Deemed University, Dental College And \\ Hospital, Pune, India) \\ ${ }_{3}^{3}$ (Department Of Oral And Maxillofacial Surgery/ Bharati Vidyapeeth Deemed University, Dental College And \\ Hospital, Pune, India) \\ ${ }^{4}$ (Department Of Oral And Maxillofacial Surgery/ Bharati Vidyapeeth Deemed University, Dental College And \\ Hospital, Pune, India)
}

\begin{abstract}
The treatment of keratocystic odontogenic tumour remains controversial. The aim of this article is to report the outcome of conservative treatment protocol for a large keratocystic odontogenic tumour crossing the midline treated with enucleation and open dressing.

A 33 year old male patient with mandibular anterior swelling was referred to Department of Oral and Maxillofacial Surgery. CBCTreports revealed a large radiolucent lesion extending from 37 to 46 region and associated with bilateral mandibular impacted canines. Histopathological evaluation suggested akeratocystic odontogenic tumour. To minimize the possibility of pathologic fracture and complex surgical intervention the patientwas treated by enucleation followed by open packing. This conservative treatmentwas selected since the patient was young. Postoperative results are encouraging.

This conservative treatment protocol for keratocystic odontogenic tumors, based on enucleation followed by open packing can be a possible choice in young patients.
\end{abstract}

Keywords: keratocysts; bilateral impacted mandibular canines; cone beam computer tomography.

\section{Introduction}

The term "odontogenic keratocyst" was first coined by Philipsen in 1956[1]. In the recent, past observations were made which showed that the odontogenic keratocyst behaved more like a neoplasm and not as a cystic lesion.In agreement with these findings, the World health organization (WHO) in 2005recommended the term keratocystic odontogenic tumor (KCOT)) for this lesion[2, 3]. It better reflects the aggressive clinical behavior, histologically high mitotic rate and its association with genetic and chromosomal abnormalities. The malignant transformation of KCOTs has also been reported[4].

Kcot are known to have recurrence rate ranging from $13 \%$ to $80 \%$ [1]. Literature suggests that the rate of recurrence is largely depending on the mode of treatment used. Treatment of KCOTs till date remains controversial and includesan entirespectrum of treatment modalities ranging from marsupialisation to segmental resection.

Literature reviews suggest that most cases recur within the first 5 years after treatment[5,6]. Many operative techniques including the use of Carnoy's solution, cryotherapy and electrocauterisation have been proposed to reduce the incidence of recurrence[5]. Although a definitiveprotocol for management of KCOTs has not been established, it would be better to use conservative treatments for the first appearances and more aggressivemeasures for recurrences. dressing.

In this article,we present a case of a large KCOT crossing the midline treatedwith enucleation and open

\section{Case report}

A 30-year-old male was reported to our institute in June 2016. The patient had visited a private dentist earlier for root canal treatment of 46 . Following completion of the root canal treatment the patient noticed a swelling in the left mandibular vestibule. Subsequently the dentist advised an OPG which revealed a large radiolucent lesion extending on either side of the midline. The dentist then referred the patient to our institute for further evaluation and treatment.

Intraorally on examination a moderate swelling was visible in the region of 34 and 35 . Overlying mucosa was normal without any evidence of sinus formation. The cone beam computer tomography (CBCT) report described the lesion as depicting two well defined, expanded, radiolucent lesions extending from 37 to 46 
mesiodistally and super inferiorly extending from middle third of their roots to inferior border of mandible with bilateral impacted canine. [Fig.1,2].

Clinically sutures were evident in the labial vestibule at the site of previous biopsy.[Fig. 3] All investigations required to ascertain fitness for the surgical procedure were performed. Medical history revealed that the patient was hypertensive and asthmatic and was on medication since 2 years. Reports revealed that the haemoglobin level was suboptimal $(7.0 \mathrm{mg} / \mathrm{dl})$. The patient was administered 3 pint PCV. The patient was posted for surgical intervention under General Anaesthesia using nasotracheal intubation. Local anaesthetic solution containing 1:200000 adrenaline wasinfiltrated inthe labial and buccal vestibule. A degloving incision was taken from 37 to 46 to provide satisfactory exposure of the lesion. Decortication of buccal cortex was done and lining of the lesion was visible[Fig.4, 5]. The cyst was enucleated completely and extraction of bilateral impacted canines was performed[Fig. 6, 7]. Sharp edges of bone were rounded off using a HP 10 SS White bur. The exposed surfaces of the bony cavity were electrocauterised to reduce the possibility of recurrence. The mucosal margins were approximated with bone and suturing was done with resorbable sutures. Open dressing soaked in povidone iodine ointment was packed into the cavity [Fig. 8]. The specimen removed was sent for histopathological diagnosis and the final diagnosis of lesion was revealed as KCOT[Fig. 9, 10].Currently the patient is under medical supervision to monitor the healingprocess [Fig.11].

\section{Discussion}

The keratocystic odontogenic tumour is derived from the remnants of the dental lamina, with a biologic behavior similar to a benign neoplasm, with a distinctive lining of six to ten in thickness, and that exhibits a basal layer of palisaded cells and a surface of corrugated parakeratin[7].

An unerupted tooth is involved in the lesion in 25 to $40 \%$. As per earlier studies, the KCOT is known to arise from dental lamina rests near an unerupted tooth and it grows to enclose the unerupted tooth[8].Epithelial islands derived from the dental lamina are mainly found in the gingiva and periodontal ligament [9].It is still not known why KCOT develop from such epithelial islands or microcyst.

Increased osmotic pressure within the lumen of the cyst leads to increase in size of most of the cysts. But this mechanism appears to be false as far as the KCOT is concerned. Factors affecting the growth of KCOT's are still unknown. These factors may be inherent in the epithelium itself or there might be enzymatic activity in the fibrous wall. The growth of KCOT is usually in anteroposterior direction causing minimal expansion of bone.

Successful treatment of KCOT remains to be controversial because of its high recurrence rate. In almost all cases, presence of microcyst or epithelial islands is noted. According to most authors, these microcysts play a crucial role in recurrences of KCOT's. Therefore it is important to eliminate all the vital cells that are left behind in the defect.

Treatment of KCOT depends on the size of the lesion and amount of cortical perforation.

There are various treatment options including marsupialisation alone, marsupialisation followed by enucleation, electrocauterisation, use of liquid nitrogen, Carnoy's solution, decompression followed by enucleation with open dressing and en bloc and segmental resection[6].

Marsupialisation is done to reduce the size of cyst and once the size of lesion is reduced, cyst is enucleated. Liquid nitrogen can also be used but its disadvantage is that its freezing is not uniform and it destroys the surrounding vital structures [10].

Studies have shown that use of Carnoy's solution reduces the recurrence rate.Carnoy's solution can be used before or after enucleation of cyst.Decompression followed by enucleation was first given by Brondum and Jensen1992 and Marker et al in 1997[11,12]. This procedure is known to be more conservative.

Block resection has proven to be the most predictable treatment but it is associated with significant morbidity. Reconstructing the continuity defects of the mandible are extremely challenging and rarely succeed in restoring presurgical anatomic and functional status [13].

In this case we decided to treat the defect by enucleating the tumour followed by open dressing.It would be prudent to note that the CBCT reported two separatelesions; however during surgical intervention we found a single lesion. Normally KCOT's have a fragile lining and it comes out in pieces while enucleating but clinically we found that the lesion had a thick lining. As we know that the eruption of mandibular permanent canines is at the age of 9 to 10 years. In this case both the canines were impacted suggesting that the lesion could have been present since childhood but remained undiagnosed. Because KCOT's grow within the medullary space of bone in anteroposterior direction, diagnosis is often delayed until the lesion has reached a formidable size.

\section{Conclusion}

Enucleation with open dressing is a recommended treatment option for large KCOT's, particularly in younger patients= Numerous conservative approaches can be used in primary stage of KCOT's. In case of 
recurrences, extensive treatment options such as marsupialisation and en block resection should be considered. Efforts should be taken by the operator to preferably enucleate the lesion in toto to reduce the chances of recurrence.

\section{References}

[1]. GülsünYildirim, HanifeAtaoglu, Abdullah Kalayci, BirkanTahaÖzkan, KorhanKucuk, AlparslanEsen, Conservative Treatment Protocol for Keratocystic Odontogenic Tumour: a Follow-up Study of 3 Cases, J Oral Maxillofac Res, 1(3), $2010,1-11$.

[2]. Philipsen HP. In: Barnes L, Eveson JW, Reichart P, et al, World Health Organization classification of tumours. Pathology and genetics of head and neck tumours. Lyon (France): IARC, 2005, 306-7.

[3]. Vedtofte P, Praetorius F, Recurrence of the odontogenic keratocyst in relation to clinical and histological features. A 20-year follow-up study of 72 patients.Int J Oral Surg, 8(6), 1979, 412-20.

[4]. Myoung H, Hong SP, Hong SD, Lee JI, Lim CY, Choung PH, Lee JH, Choi JY, Seo BM, Kim MJ, Odontogenic keratocyst: Review of 256 cases for recurrence and clinicopathologic parameters. Oral Surg Oral Med Oral Pathol Oral RadiolEndod, 91(3), 2001, 328-33.

[5]. Forssell K, Forssell H, Kahnberg KE. Recurrenceof keratocysts.A long-term follow-up study.Int J Oral MaxillofacSurg, 17(1), 1988, 25-8.

[6]. Paul J.W. Stoelinga, The Treatment of Odontogenic Keratocysts by Excision of the Overlying,Attached Mucosa, Enucleation, and Treatment of the Bony Defect With Carnoy Solution J Oral MaxillofacSurg, 63, 2005,1662-6.

[7]. MrRajendran and Sivapathasundharam, Shafer's Textbook of Oral Pathology (New Delhi, Elsevier,2005). (5).

[8]. Paolo Boffano, EmanueleRuga, CesareGallesio, KeratocysticOdontogenic Tumor(Odontogenic Keratocyst): PreliminaryRetrospective Review of Epidemiologic,Clinical, and Radiologic Features of 261Lesions From University of Turin, American Association of Oral and Maxillofacial Surgeons, 2010.

[9]. Paul J.W. Stoelinga, Etiology and pathogenesis of keratocystsDepartment of Oral and Maxillofacial Surgery, University Hospital, UMC St Radboud 421, University of Nijmegen,

[10]. 6500 HB Nijmegen, The Netherlands Clin N Am, 15, 2003, 317-324.

[11]. Brian L. Schmidt, The use of liquid nitrogen cryotherapy in the management of the odontogenic keratocyst,15, 2003, 393-405.

[12]. Worrall SF, Recurrent odontogenic keratocyst within the temporalis muscle. Br J Oral MaxillofacSurg, 30(1), 1992, 59-62.

[13]. Browne RM, The odontogenic keratocyst. Histological features and their correlation with clinical behaviour. Br Dent J, 131(6), 1971, 249-59.

[14]. R. Bryan Bell, Eric J. Dierks, Treatment options for the recurrent odontogenic keratocyst, 15, 2003, $429-446$.
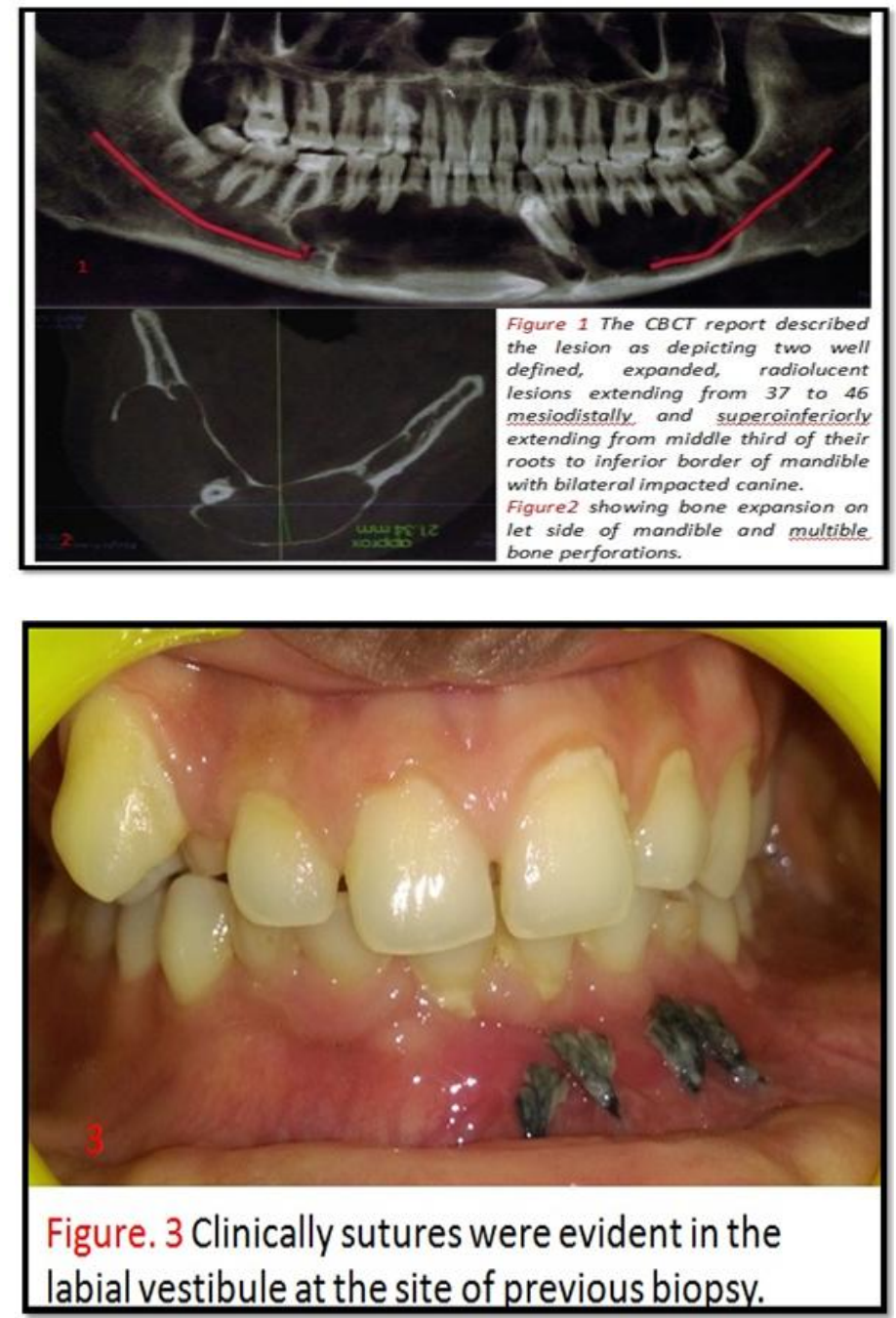

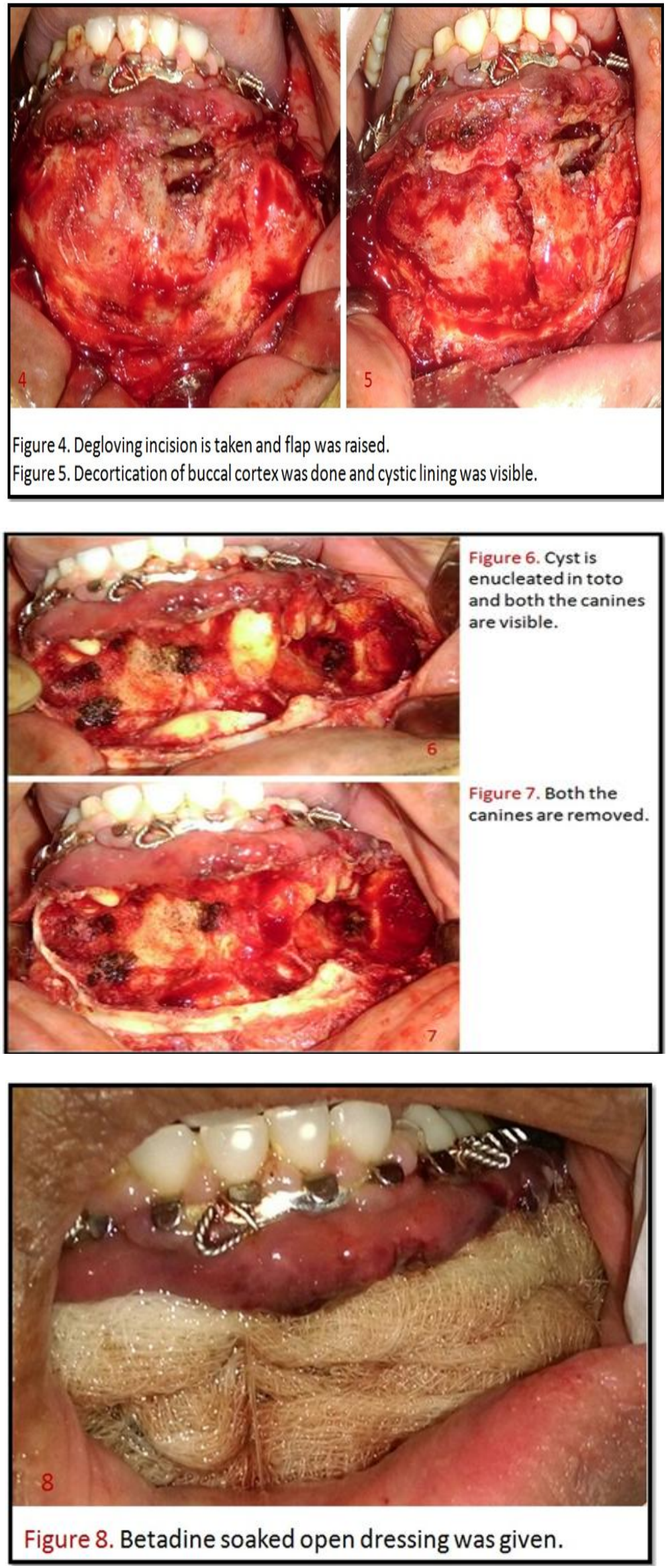

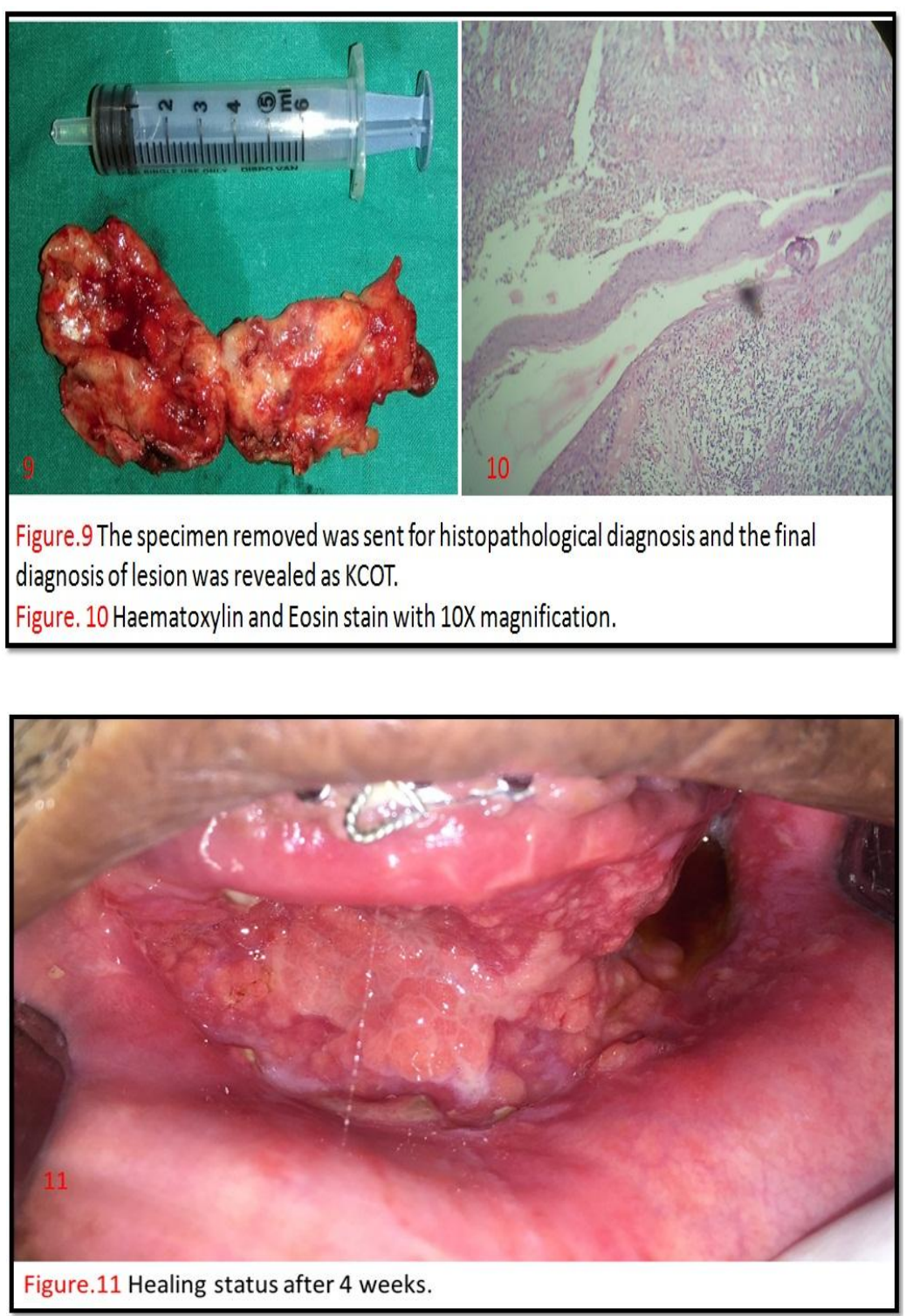